\title{
Exotics at Our Home
}

\author{
Nikolay Achasov ${ }^{1, *}$ \\ ${ }^{1}$ Sobolev Institute for Mathematics, 630090 Novosibirsk, Russia
}

\begin{abstract}
.
1. Light Scalars as Four-Quark States

2. Isotensor Tensor $E(1500-1600)$ State

3. $X(3872)$ State as Charmonium $\chi_{c 1}(2 P)$

4. Two-gluon Annihilation of Charmonium $\chi_{c 2}(2 P)$
\end{abstract}

\section{Introduction}

I would like to discuss the exotic phenomena that I know not by hearsay. That is, the exotic phenomena, which we analyzed, explained and made the new predictions, the considerable part of which is confirmed already by experiment, and other ones wait the experimental checking.

\section{Light Scalars as Four-quark States}

The $a_{0}(980)$ and $f_{0}(980)$ mesons are well-established parts of the proposed light scalar meson nonet [1]. From the beginning, the $a_{0}(980)$ and $f_{0}(980)$ mesons became one of the central problems of nonperturbative QCD, as they are important for understanding the way chiral symmetry is realized in the low-energy region and, consequently, for understanding confinement. Many experimental and theoretical papers have been devoted to this subject.

There is much evidence that supports the four-quark model of light scalar mesons [2, 3].

The suppression of the $a_{0}^{0}(980)$ and $f_{0}(980)$ resonances in the $\gamma \gamma \rightarrow \eta \pi^{0}$ and $\gamma \gamma \rightarrow \pi \pi$ reactions, respectively, was predicted in 1982 [4], $\Gamma_{a_{0}^{0} \gamma \gamma} \approx \Gamma_{f_{0} \gamma \gamma} \approx 0.27 \mathrm{keV}$, and confirmed by experiment [1]. The high quality Belle data $[5,6]$ allowed to elucidate the mechanisms of the $\sigma(600), f_{0}(980)$, and $a_{0}^{0}(980)$ resonance production in $\gamma \gamma$ collisions confirmed their four-quark structure $[7,8]$. Light scalar mesons are produced in $\gamma \gamma$ collisions mainly via rescatterings, that is, via the four-quark transitions. As for $a_{2}(1320)$ and $f_{2}(1270)$ (the wellknown $q \bar{q}$ states), they are produced mainly via the two-quark transitions (direct couplings with $\gamma \gamma$ ). As a result the practically model-independent prediction of the $q \bar{q}$ model $g_{f_{2} \gamma \gamma}^{2}$ : $g_{a_{2} \gamma \gamma}^{2}=25: 9$ agrees with experiment rather well. As to the ideal $q \bar{q}$ model prediction $g_{f_{0} \gamma \gamma}^{2}: g_{a_{0} \gamma \gamma}^{2}=25: 9$, it is excluded by experiment.

The argument in favor of the four-quark nature of $a_{0}(980)$ and $f_{0}(980)$ is the fact that the $\phi(1020) \rightarrow a_{0}^{0} \gamma$ and $\phi(1020) \rightarrow f_{0} \gamma$ decays go through the kaon loop: $\phi \rightarrow K^{+} K^{-} \rightarrow a_{0}^{0} \gamma$,

\footnotetext{
*e-mail: achasov@math.nsc.ru
} 
$\phi \rightarrow K^{+} K^{-} \rightarrow f_{0} \gamma$, i.e., via the four-quark transition [9-13]. The kaon-loop model was suggested in Ref. [9] and confirmed by experiment ten years later [14-16].

It was shown in Ref. [10] that the production of $a_{0}^{0}(980)$ and $f_{0}(980)$ in $\phi \rightarrow a_{0}^{0} \gamma \rightarrow \eta \pi^{0} \gamma$ and $\phi \rightarrow f_{0} \gamma \rightarrow \pi^{0} \pi^{0} \gamma$ decays is caused by the four-quark transitions, resulting in strong restrictions on the large- $N_{C}$ expansion of the decay amplitudes. The analysis showed that these constraints give new evidence in favor of the four-quark nature of the $a_{0}(980)$ and $f_{0}(980)$ mesons.

In Refs. $[17,18]$ it was shown that the description of the $\phi \rightarrow K^{+} K^{-} \rightarrow \gamma a_{0}^{0}(980) / f_{0}(980)$ decays requires virtual momenta of $K(\bar{K})$ greater than $2 \mathrm{GeV}$, while in the case of loose molecules with a binding energy about $20 \mathrm{MeV}$, they would have to be about $100 \mathrm{MeV}$. Besides, it should be noted that the production of scalar mesons in the pion-nucleon collisions with large momentum transfers also points to their compactness [19].

It was also shown in Refs. [20, 21] that the linear $S_{L}(2) \times S_{R}(2) \sigma$ model [22] reflects all of the main features of low-energy $\pi \pi \rightarrow \pi \pi$ and $\gamma \gamma \rightarrow \pi \pi$ reactions up to energy $0.8 \mathrm{GeV}$ and agrees with the four-quark nature of the $\sigma$ meson. This allowed for the development of a phenomenological model with the right analytical properties in the complex $s$ plane that took into account the linear $\sigma$ model, the $\sigma(600)-f_{0}(980)$ mixing and the background [23]. This background has a left cut inspired by crossing symmetry, and the resulting amplitude agrees with results obtained using the chiral expansion, dispersion relations, and the Roy equation [24], and with the four-quark nature of the $\sigma(600)$ and $f_{0}(980)$ mesons as well. This model well describes the experimental data on $\pi \pi \rightarrow \pi \pi$ scattering up to $1.2 \mathrm{GeV}$.

Moreover, the absence of $J / \psi \rightarrow \gamma f_{0}(980), \rho a_{0}(980), \omega f_{0}(980)$ decays in the presence of intense $J / \psi \rightarrow \gamma f_{2}(1270), \gamma f_{2}^{\prime}(1525), \rho a_{2}(1320), \omega f_{2}(1270)$ decays is at variance with the $P$-wave two-quark, $q \bar{q}$, structure of $a_{0}(980)$ and $f_{0}(980)$ resonances [25].

It is shown in Ref. [26] that the recent data on the $K_{S}^{0} K^{+}$correlation in $\mathrm{Pb}-\mathrm{Pb}$ interactions Ref. [27] agree with the data on the $\gamma \gamma \rightarrow \eta \pi^{0}$ and $\phi \rightarrow \eta \pi^{0} \gamma$ reactions and support the fourquark model of the $a_{0}(980)$ meson. It is shown that the data does not contradict the validity of the Gaussian assumption.

In Refs. [28, 29] it was suggested the program of studying light scalars in semileptonic $D$ and $B$ decays, which are the unique probe of the $q \bar{q}$ constituent pair in the light scalars. We studied production of scalars $\sigma(600)$ and $f_{0}(980)$ in the $D_{s}^{+} \rightarrow s \bar{s} e^{+} v \rightarrow \pi^{+} \pi^{-} e^{+} v$ decays, the conclusion was that the fraction of the $s \bar{s}$ constituent components in $\sigma(600)$ and $f_{0}(980)$ is small. Unfortunately, at the moment the CLEO statistics [30] is rather poor, and thus new high-statistics data are highly desirable.

It was noted in Refs. [28, 29] that no less interesting is the study of semileptonic decays of $D^{0}$ and $D^{+}$mesons $-D^{+} \rightarrow d \bar{d} e^{+} v \rightarrow\left[\sigma(600)+f_{0}(980)\right] e^{+} v \rightarrow \pi^{+} \pi^{-} e^{+} v$, $D^{0} \rightarrow d \bar{u} e^{+} v \rightarrow a_{0}^{-} e^{+} v \rightarrow \pi^{-} \eta e^{+} v$ and $D^{+} \rightarrow d \bar{d} e^{+} v \rightarrow a_{0}^{0} e^{+} v \rightarrow \pi^{0} \eta e^{+} v$ (or the chargedconjugated ones) which had not been investigated. It is very tempting to study light scalar mesons in semileptonic decays of $B$ mesons [29]: $B^{0} \rightarrow d \bar{u} e^{+} v \rightarrow a_{0}^{-} e^{+} v \rightarrow \pi^{-} \eta e^{+} v$, $B^{+} \rightarrow u \bar{u} e^{+} v \rightarrow a_{0}^{0} e^{+} v \rightarrow \pi^{0} \eta e^{+} v, B^{+} \rightarrow u \bar{u} e^{+} v \rightarrow\left[\sigma(600)+f_{0}(980)\right] e^{+} v \rightarrow \pi^{+} \pi^{-} e^{+} v$.

Recently BESIII Collaboration measured the decays $D^{0} \rightarrow d \bar{u} e^{+} v \rightarrow a_{0}^{-} e^{+} v \rightarrow \pi^{-} \eta e^{+} v$ and $D^{+} \rightarrow d \bar{d} e^{+} v \rightarrow a_{0}^{0} e^{+} v \rightarrow \pi^{0} \eta e^{+} v$ for the first time [31]. In Ref. [32] we discuss the Ref. [28] program in light of these measurements taking into account contribution of $a_{0}^{\prime}$ meson with mass about $1400 \mathrm{MeV}$. A variant when $a_{0}^{-}(980)$ has no $q \bar{q}$ constituent component at all is presented in Figure 1, that is, $a_{0}^{-}(980)$ is produced as a result of mixing $a_{0}^{\prime-}(1400) \rightarrow a_{0}^{-}(980)$, $D^{0} \rightarrow d \bar{u} e^{+} v \rightarrow a_{0}^{\prime-} e^{+} v \rightarrow a_{0}^{-} e^{+} v \rightarrow \pi^{-} \eta e^{+} v$

The first measurement of BESIII is the important step for the investigation of light scalar mesons nature, but for the present the statistics is not adequate for the conclusions. 


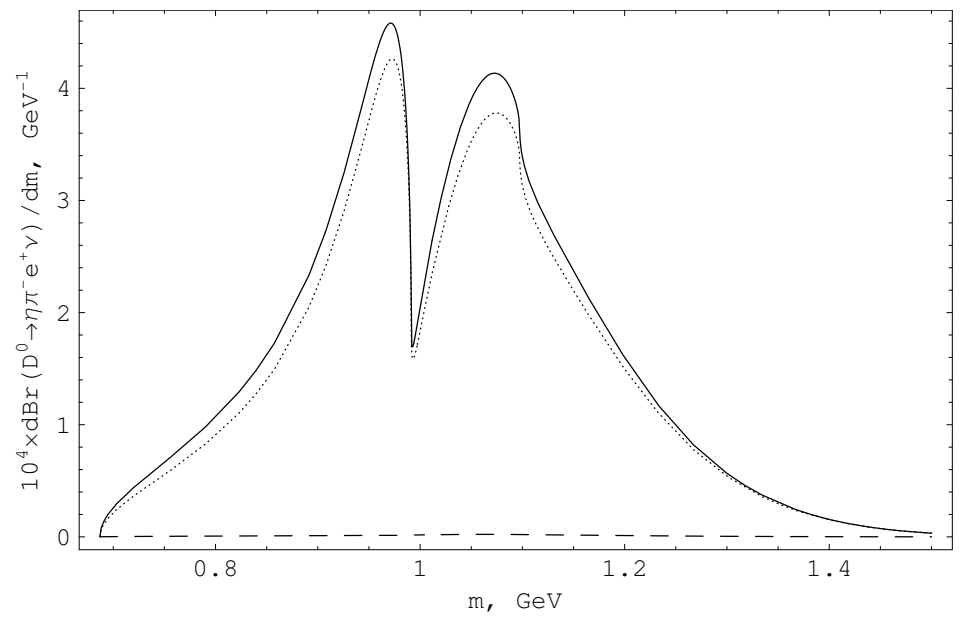

Figure 1. The plot of $D^{0} \rightarrow\left(a_{0}^{-}, a_{0}^{\prime-}\right) e^{+} v \rightarrow \eta \pi^{-} e^{+} v$ spectrum without a coupling of $a_{0}^{-}(980)$ with the constituent $d \bar{u}$ state. The solid line is the total contribution, the dotted line is caused by the mixing $a_{0}^{\prime-}(1400) \rightarrow a_{0}^{-}(980)$, the dashed line is caused by the $a_{0}^{\prime-}(1400)$ production, Ref. [32].

\section{Isotensor Tensor $E(1500-1600)$ State}

Thirty six years ago we predicted [4] the striking interference picture in the $\gamma \gamma \rightarrow \rho^{0} \rho^{0}$ and $\gamma \gamma \rightarrow \rho^{+} \rho^{-}$reactions in the $q^{2} \bar{q}^{2}$ MIT model [2].

We explained the strong boost near the threshold in the $\gamma \gamma \rightarrow \rho^{0} \rho^{0}$ reaction by the production of the isotensor tensor and isoscalar tensor resonances, then the destructive interference of their contributions in the $\gamma \gamma \rightarrow \rho^{+} \rho^{-}$reaction follows from isotopic symmetry!

Experiment backed up this prediction, JADE 1983, ARGUS 1991, see Figure 2 and Refs. $[33,34]$.

We believe that the Belle data will support the above picture.

We [35] hope that JEFLAB will find the charged components of the isotensor tensor state $E^{ \pm}$in the mass spectra of the $\rho^{ \pm} \rho^{0}$ states in the reactions $\gamma N \rightarrow \rho^{ \pm} \rho^{0} N(\Delta)$.

We $[34,36]$ believe also that IHEP in Protvino could find $E^{ \pm \pm}$in the mass spectra of the $\rho^{ \pm} \rho^{ \pm}$states in the reactions $\pi N \rightarrow \pi \rho^{ \pm} \rho^{ \pm} N(\Delta)$ and $N N \rightarrow N(\Delta) \rho^{ \pm} \rho^{ \pm} N(\Delta)$.

\section{$4 X(3872)$ State as Charmonium $\chi_{c 1}(2 P)$, Refs. [37-39]}

The two dramatic discoveries have generated a stream of the $D^{* 0} \bar{D}^{0}+D^{0} \bar{D}^{* 0}$ molecular interpretations of the $X(3872)$ resonance. The mass of the $X(3872)$ resonance is $50 \mathrm{MeV}$ lower than predictions of the most lucky naive potential models for the mass of the $\chi_{c 1}(2 P)$ resonance,

$$
m_{X}-m_{\chi_{c 1}(2 P)}=-\Delta \approx-50 \mathrm{MeV}
$$

and the relation between the branching ratios

$$
B R\left(X \rightarrow \pi^{+} \pi^{-} \pi^{0} J / \psi(1 S)\right) \sim B R\left(X \rightarrow \pi^{+} \pi^{-} J / \psi(1 S)\right),
$$

that is interpreted as a strong violation of isotopic symmetry. 


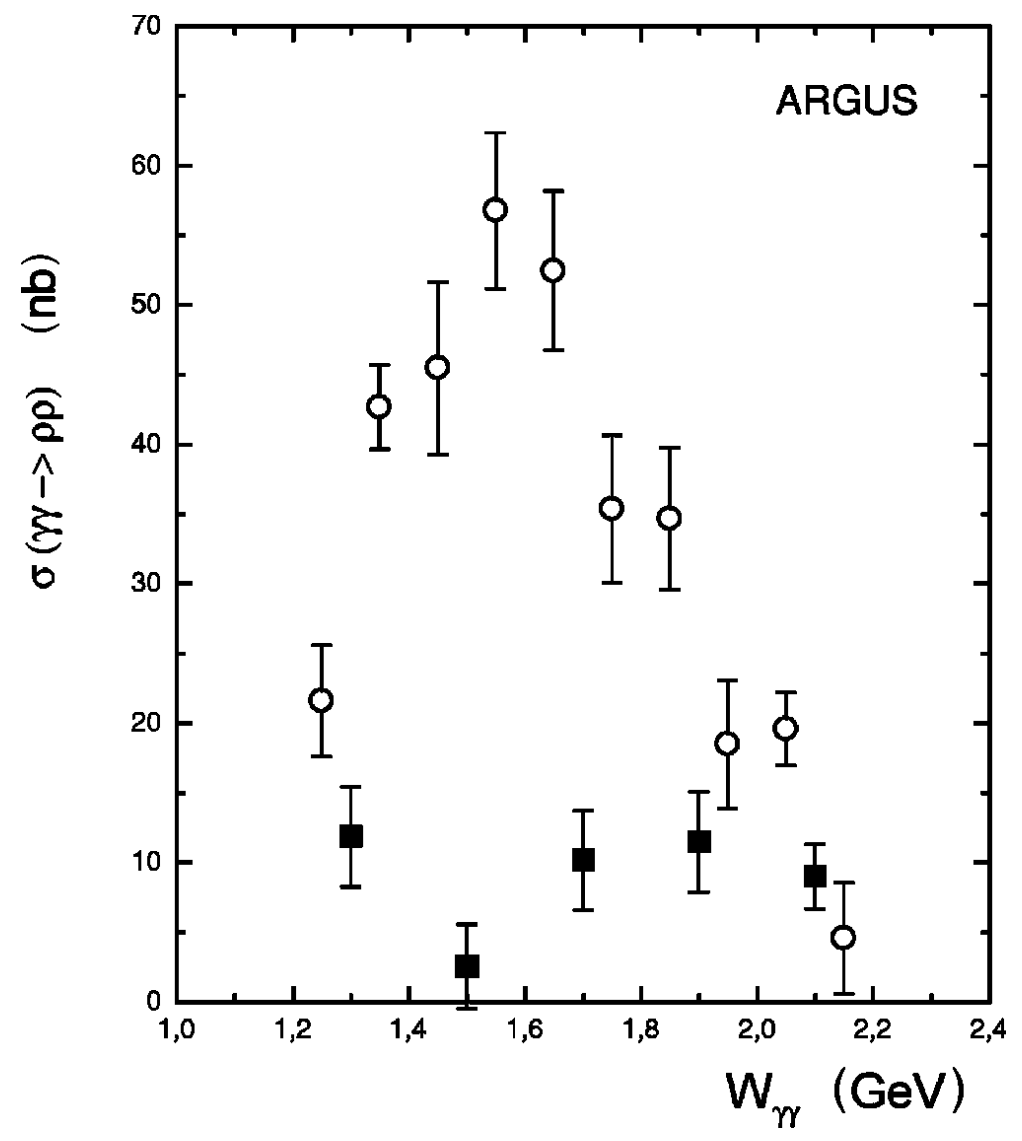

Figure 2. The white circles are $\gamma \gamma \rightarrow \rho^{0} \rho^{0}$; the black small squares are $\gamma \gamma \rightarrow \rho^{+} \rho^{-}$.

But, the bounding energy is small, $\epsilon_{B} \lesssim 1 \mathrm{MeV}$. That is, the radius of the molecule is large, $r_{X(3872)} \gtrsim 5=5 \cdot 10^{-13} \mathrm{~cm}$. As for the charmonium, its radius is less one fermi, $r_{\chi_{c 1}(2 P)} \lesssim$ fermi $=10^{-13} \mathrm{~cm}$.

That is, the molecule volume is $125 \div 1000$ times as large as the charmonium volume, $V_{X(3872)} / V_{\chi_{c 1}(2 P)} \gtrsim 125 \div 1000$. This means a probability of production of a giant molecule in hard processes, at small distances, is suppressed in comparison with a probability of production of heavy a charmonium by a factor $\sim V_{\chi_{c 1}(2 P)} / V_{X(3872)}$.

But, in reality [1, 38-41]

$$
0.74<\frac{\sigma(p p \rightarrow X(3872)+\text { anything })}{\sigma(p p \rightarrow \psi(2 S)+\text { anything })}<2.1 .
$$

with rapidity in the range $2,5-4,5$ and transverse momentum in the range $5-20 \mathrm{GeV}$.

In addition, [1, 39, 41, 43]

$$
0.2<\frac{B R\left(B^{0} \rightarrow X(3872) K^{+} \pi^{-}\right)}{B R\left(B^{0} \rightarrow \psi(2 S) K^{+} \pi^{-}\right)}<0.6
$$

The extended molecule is produced in hard processes as intensively as the compact charmonium. It's miracle! 
We explain the shift of the mass of the $X(3872)$ resonance with respect to the prediction of a potential model for the mass of the $\chi_{c 1}(2 P)$ charmonium by the contribution of the virtual $D^{*} \bar{D}+$ c.c. intermediate states into the self energy of the $X(3872)$ resonance $[38,39]$.

This allows us to estimate the coupling constant of the $X(7872)$ resonance with the $D^{* 0} \bar{D}^{0}$ channel, the branching ratio of the $X(3872) \rightarrow D^{* 0} \bar{D}^{0}+$ c.c. decay, and the branching ratio of the $X(3872)$ decay into all non $-D^{* 0} \bar{D}^{0}+$ c.c. states [37-39].

We $[38,39]$ predict that the hadron channels of the decays of $\chi_{c 1}(2 P)$ via two gluon ( $X(3872) \rightarrow$ gluon gluon $\rightarrow$ hadrons $)$ should be the same as in the $\chi_{c 1}(1 P)$ case, that is, there should be a few tens of such channels. The discovery of these decays would be the strong (if not decisive) confirmation of our scenario.

As for $B R(X \rightarrow \omega J / \psi) \sim B R(X \rightarrow \rho J / \psi)$, this could be a result of dynamics. In our scenario the $\omega J / \psi$ state is produced via the three gluons.

As for the $\rho J / \psi$ state, it is produced both via the one photon, and via the three gluons (via the contribution $\sim m_{u}-m_{d}$ ).

Close to our scenario is an example of the $J / \psi \rightarrow \rho \eta^{\prime}$ and $J / \psi \rightarrow \omega \eta^{\prime}$ decays. According to Ref. [1]

$$
B R\left(J / \psi \rightarrow \rho \eta^{\prime}\right)=(1.05 \pm 0.18) \cdot 10^{-4} \text { and } B R\left(J / \psi \rightarrow \omega \eta^{\prime}\right)=(1.82 \pm 0.21) \cdot 10^{-4}
$$

Note that in the $X(3872)$ case the $\omega$ meson is produced on its tail $\left(m_{X}-m_{J / \psi}=775 \mathrm{MeV}\right)$, while the $\rho$ meson is produced on a half.

It is well known that the physics of charmonium $(c \bar{c})$ and bottomonium $(b \bar{b})$ is similar. Let us compare the already known features of X(3872) with the ones of $\Upsilon_{b 1}(2 P)$.

The LHCb Collaboration published a landmark result [42]

$$
\frac{B R(X \rightarrow \gamma \psi(2 S))}{B R(X \rightarrow \gamma J / \psi)}=C_{X}\left(\frac{\omega_{\psi(2 S)}}{\omega_{J / \psi}}\right)^{3}=2.46 \pm 0.7
$$

where $\omega_{\psi(2 S)}$ and $\omega_{J / \psi}$ are the energies of the photons in the $X \rightarrow \gamma \psi(2 S)$ and $B R(X \rightarrow \gamma J / \psi)$ decays, respectively.

On the other hand, it is known [1] that

$$
\frac{B R\left(\chi_{b 1}(2 P) \rightarrow \gamma^{\Upsilon}(2 S)\right)}{B R\left(\chi_{b 1}(2 P) \rightarrow \gamma^{\Upsilon}(1 S)\right)}=C_{\chi_{b 1}(2 P)}\left(\frac{\omega_{\Upsilon(2 S)}}{\omega_{\Upsilon(1 S)}}\right)^{3}=2.16 \pm 0.28
$$

where $\omega_{\Upsilon(2 S)}$ and $\omega_{\Upsilon(1 S)}$ are the energies of the photons in the $\chi_{b 1}(2 P) \rightarrow \gamma \Upsilon \Upsilon(2 S)$ and $\chi_{b 1}(2 P) \rightarrow \gamma^{\Upsilon} \Upsilon(1 S)$ decays, respectively.

Consequently,

$$
C_{X}=136.78 \pm 38.89
$$

and

$$
C_{\chi_{b 1}(2 P)}=80 \pm 10.37
$$

as all most lucky versions of the potential model predict for the quarkonia, $C_{\chi_{c 1}(2 P)} \gg 1$ and $C_{\chi b 1}(2 P) \gg 1$.

According to Ref. [1]

$$
B R\left(\chi_{b 1}(2 P) \rightarrow \omega \Upsilon(1 S)\right)=\left(1.63 \pm_{0.34}^{0.4}\right) \%
$$

If the one-photon mechanism dominates in the $X(3872) \rightarrow \rho J / \psi$ decay then one should expect

$$
B R\left(\chi_{b 1}(2 P) \rightarrow \rho \Upsilon(1 S)\right) \sim\left(e_{b} / e_{c}\right)^{2} \cdot 1.6 \%=(1 / 4) \cdot 1.6 \%=0.4 \%,
$$


where $e_{c}$ and $e_{b}$ are the charges of the $c$ and $b$ quarks, respectively.

If the three-gluon mechanism (its part $\sim m_{u}-m_{d}$ ) dominates in the $X(3872) \rightarrow \rho J / \psi$ decay then one should expect

$$
B R\left(\chi_{b 1}(2 P) \rightarrow \rho \Upsilon(1 S)\right) \sim 1.6 \%
$$

We believe that discovery of a significant number of unknown decays of $X(3872)$ into non- $D^{* 0} \bar{D}^{0}+$ c.c. states via two gluons and discovery of the $\chi_{b 1}(2 P) \rightarrow \rho \Upsilon(1 S)$ decay could decide destiny of $X(3872)$.

Once more, we discuss the scenario where the $\chi_{c 1}(2 P)$ charmonium sits on the $D^{* 0} \bar{D}^{0}$ threshold but not a mixing of the giant $D^{*} \bar{D}$ molecule and the compact $\chi_{c 1}(2 P)$ charmonium. Note that the mixing of such states requests the special justification. That is, it is necessary to show that the transition of the giant molecule into the compact charmonium is considerable at insignificant overlapping of their wave functions. Such a transition $\sim \sqrt{V_{\chi_{c 1}(2 P)} / V_{X(3872)}}$ and a branching ratio of a decay via such a transition $\sim V_{\chi_{c 1}(2 P)} / V_{X(3872)}$.

Note that now the $X(3872)$ state is named in Ref. [1] as $\chi_{c 1}(3872)$.

The above scenario can be checked in the process $e^{+} e^{-} \rightarrow \psi(4040) \rightarrow \gamma($ gluon gluon $) \rightarrow$ $\gamma \chi_{c 1}(3872) \rightarrow \gamma($ gluon gluon $) \rightarrow \gamma$ (light hadrons) on BES III, for example, or on the super c-tau factory that is projecting in Novosibirsk.

\section{Two-gluon Annihilation of Charmonium $\chi_{c 2}(2 P)$}

We [44] expect that $B R\left(\chi_{c 2}(2 P) \rightarrow\right.$ gluon gluon $) \gtrsim 2 \%$ if the Particle Data Group as well as the BaBar and Belle collaborations have correctly identified the state.

In reality, this branching ratio corresponds to the one for $\chi_{c 2}(2 P)$ decaying into light hadrons. The hadron channels of the two-gluon decays of $\chi_{c 2}(2 P)$ should be the same as in the $\chi_{c 2}(1 P)$ case, that is, there should be a few tens of such channels.

The ratio of the two-photon and two-gluon widths of the charmonium decays does not depend on the wave function in the nonrelativistic potential model of charmonium [45]. It allows to find the low limit of $B R\left(\chi_{c 2}(2 P) \rightarrow\right.$ gluon gluon). The comparison with the wellknown data about $\chi_{c 2}(1 P)$ allows us to conclude that $B R\left(\chi_{c 2}(2 P) \rightarrow 2 g\right) \approx(6.5 \pm 2.0) \%$ is very likely.

The confirmation of the $\chi_{c 2}(2 P)$ state can be tested by BESIII, for example, through the process $e^{+} e^{-} \rightarrow \psi(4040) \rightarrow \gamma \chi_{c 2}(2 P)$. The search for two-gluon decays of the $\chi_{c 2}(2 P)$ state is feasible for BESIII as well as other super factories such as BaBar and Belle.

Note that now the $\chi_{c 2}(2 P)$ state is named in Ref. [1] as $\chi_{c 2}(3930)$.

\section{Acknowledgments}

I am grateful to Organizers of QUARKS-2018 for the kind Invitation.

The work was supported by the program of fundamental scientific researches of the SB RAS No. II.15.1., project No. 0314-2016-0021 and partially by the Russian Foundation for Basic Research Grant No. 16-02-00065.

\section{References}

[1] M. Tanabashi et al. (Particle Data Group), Phys. Rev. D 98, 030001 (2018).

[2] R.L. Jaffe, Phys. Rev. D 15, 267 (1977);

Phys. Rev. D 15, 281 (1977). 
[3] S. Weinberg, Phys. Rev. Lett. 110, 261601 (2013).

[4] N.N. Achasov, S.A. Devyanin, and G.N. Shestakov, Phys. Lett. B 108, 134 (1982);

Z. Phys. C 16, 55 (1982).

[5] S. Uehara et al. (Belle Collaboration), Phys. Rev. D 78, 052004 (2008).

[6] S. Uehara et al. (Belle Collaboration), Phys. Rev. D 80, 032001 (2009).

[7] N.N. Achasov and G.N. Shestakov, Z. Phys. C 41, 309 (1988).

[8] N.N. Achasov and G.N. Shestakov, Phys. Rev. D 77, 074020 (2008);

Phys. Rev. D 81, 094029 (2010);

Usp. Fiz. Nauk 54, 799 (2011) [Sov. Phys. Usp. 181, 827 (2011)].

[9] N.N. Achasov and V.N. Ivanchenko, Nucl. Phys. B 315, 465 (1989).

[10] N.N. Achasov, Nucl. Phys. A 728, 425 (2003).

[11] N.N. Achasov and V.V. Gubin, Phys. Rev. D 63, 094007 (2001);

N.N. Achasov and A.V. Kiselev, Phys. Rev. D 73, 054029 (2006).

[12] N.N. Achasov and A.V. Kiselev, Phys. Rev. D 68, 014006 (2003).

[13] N.N. Achasov and V.V. Gubin, Phys. Rev. D 56, 4084 (1997).

[14] M.N. Achasov et al. (SND Collaboration), Phys. Lett. B 438, 441 (1998);

M.N. Achasov et al., Phys. Lett. B 479, 53 (2000).

[15] M.N. Achasov et al. (SND Collaboration), Phys. Lett. B 440, 442 (1998);

M.N.Achasov et al., Phys. Lett. B 485, 349 (2000);

R.R. Akhmetshin et al. (CMD-2 Collaboration) Phys. Lett. B 462, 380 (1999);

A.Aloisio et al. (KLOE Collaboration) Phys. Lett. B 537, 21 (2002);

C. Bini, P. Gauzzi, S. Giovanella, D. Leone, and S. Miscetti, KLOE Note 173 06/02, http://www.lnf.infn.it/kloe/.

[16] A.Aloisio et al. (KLOE Collaboration) Phys. Lett. B 536, 209 (2002).

[17] N.N. Achasov, V.V. Gubin, and V.I. Shevchenko, Phys. Rev. D 56, 203 (1997).

[18] N.N. Achasov and A.V. Kiselev, Phys. Rev. D 76, 077501 (2007);

Phys. Rev. D 78, 058502 (2008).

[19] N.N. Achasov and G.N. Shestakov, Phys. Rev. D 58, 054011 (1998).

[20] N.N. Achasov and G.N. Shestakov, Phys. Rev. D 49, 5779 (1994).

[21] N.N. Achasov and G.N. Shestakov, Phys. Rev. Lett. 99, 072001 (2007).

[22] M. Gell-Mann and M. Levy, Nuovo Cimento 16, 705 (1960).

[23] N.N. Achasov and A.V. Kiselev, Phys. Rev. D 83, 054008 (2011);

Phys. Rev. D 85, 094016 (2012).

[24] I. Caprini, G. Colangelo and H. Leutwyler, Phys. Rev. Lett. 96, 132001 (2006).

[25] N.N. Achasov, Usp. Fiz. Nauk 41, 1257 (1998) [Phys. Usp. 41, 1149 (1998)];

Yad. Fiz. 65, 573 (2002) [Phys. At. Nucl. 65, 546 (2002)].

[26] N.N. Achasov and A.V. Kiselev, Phys. Rev. D 97, 036015 (2018).

[27] S. Acharya et al. (ALICE Collaboration), Phys. Lett. B 774, 64 (2017).

[28] N.N. Achasov and A.V. Kiselev, Phys. Rev. D 86, 114010 (2012).

[29] N.N. Achasov and A.V. Kiselev, Int. J. Mod. Phys. Conf. Ser. 35, 1460447 (2014),

http://www.worldscientific.com/doi/pdf/10.1142/S2010194514604475.

[30] K.M Ecklund et al. CLEO Collaboration, Phys. Rev. D 80, 052009 (2009).

[31] M. Ablikim et al. (BESIII Collaboration), arXiv:1803.02166.

[32] N.N. Achasov and A.V. Kiselev, arXiv:1805.10145.

[33] N.N. Achasov and G.N. Shestakov, Z. Phys. C 27, 99 (1985).

[34] N.N. Achasov and G.N. Shestakov, Sov. Phys. Usp. 34 (6), 471 (1991).

[35] N.N. Achasov and G.N. Shestakov, Phys. Rev. D 60, 114021 (1999). 
[36] N.N. Achasov and G.N. Shestakov, Int. J. Mod. Phys. A 74313 (1992).

[37] N.N. Achasov and E.V. Rogozina, JETP Lett. 100, 227 (2014).

[38] N.N. Achasov and E.V. Rogozina, Mod. Phys. Lett. A 30, 1550181 (2015);

J.Univ.Sci.Tech.China 46, 574 (2016).

[39] Nikolay Achasov, EPJ Web Conf. 125, 04002 (2016);

N.N. Achasov, Phys. Part. Nucl. 48, 839 (2017).

[40] R. Aaij et al. (LHCb Collaboration), Eur. Phys.J. C 72, 1972 (2012).

[41] C.-Z. Yuan (Belle Collaboration), Proceedings of the XXIX PHYSICS IN COLLISION

Kobe, Japan, arXiv: 0910.3138 [hep-ex] (2009)

[42] R. Aaij et al. (LHCb Collaboration), Nucl. Phys. B 886, 665 (2014).

[43] A. Bala, et al. (Belle Callaboration), Phys. Rev. D 91 051101(R) (2015)

[44] N.N. Achasov and Kang Xian-Wei, Chin. Phys. C 41, 123102 (2017).

[45] V.A. Novikov, L.B. Okun, M.A. Shifman, A.I. Vainshtein, M.B. Voloshin, and

V.I. Zakharov, Phys. Rept. 41, 1 (1978). 